\title{
Diseño y validación de una escala basada en el concepto PIRO, una aproximación empírica a un modelo de pronóstico de los pacientes con sepsis
}

\author{
Design and validation of a scale based on the concept PIRO, an empirical approximation of a model of \\ prognosis of patients with sepsis
}

\section{María Eugenia Niño-Mantilla'}

1 Médico y cirujano, Msc. Epidemiología. Investigadora Universidad Autónoma de Bucaramanga. Floridablanca - Santander, Colombia. e-mail: mnino@unab.edu.co pronóstico de los pacientes con sepsis. Univ. Salud. 2016;18(2):214-218. DOI: http://dx.doi.org/10.22267/rus.161802.32

\begin{abstract}
Resumen
Introducción: La sepsis es un síndrome de respuesta inflamatoria sistémica secundaria a la infección, la cual ocasiona entre un 40\%-50\% de los casos de muerte en unidades de cuidado intensivo, el concepto PIRO fue propuesto como un nuevo elemento de clasificación de la sepsis. Objetivo: Analizar algunos elementos correspondientes a esta clasificación en relación con la supervivencia de los participantes en los siguientes 28 días posteriores al egreso. Materiales y métodos: Se analizaron 559 pacientes con diagnóstico de sepsis, sepsis severa y choque séptico, a los cuales se les realizó una evaluación basal de las escalas Apache II, Charlson, SOFA, edad, leucocitos y niveles de PCR durante el primer día del ingreso, adicionalmente se realizaron evaluaciones a los 28 días del egreso. Se realizó un análisis log binomial de estos predictores y se asignó un puntaje a aquellos que presentaran una asociación estadísticamente significativa. Esta escala fue comparada mediante una curva ROC con la mortalidad a los 28 días del egreso hospitalario. Resultados: La edad, la raza, el género fueron incluidas en el análisis como variables índices de predisposición (P), el sitio de infección (I) los niveles de leucocitos y PCR (R ) como variable de respuesta y la clasificación de sepsis, sepsis severa y choque séptico como variable de resultado (0). Conclusiones: La escala basada en el concepto PIRO aplicada a población colombiana, presenta puntajes relacionados con un área bajo la curva ROC de 0.75 lo cual la clasifica como una escala aceptable para evaluar a los pacientes con riesgo de mortalidad.
\end{abstract}

Palabras clave: Sepsis; infección; validez; choque séptico. (Fuente: DeCS, Bireme).

\begin{abstract}
Introduction: Sepsis is a syndrome of secondary systemic inflammatory response to infection, which causes between $40 \%-50 \%$ of cases of death in intensive care units. The PIRO concept was proposed as a new element of classification of sepsis. Objective: To analyze some relevant elements to this classification in relation to the survival of the participants in the following 28 days after discharge. Materials and methods: 559 patients diagnosed with sepsis, severe sepsis and septic shock, who underwent a baseline evaluation of the APACHE II, Charlson, SOFA scale, age, leukocytes and CRP levels during the first day of admission were analyzed; additionally, assessments were done after 28 days of discharge. A binomial log analysis of these predictors was performed and a score was assigned to those who submit a statistically significant association. This scale was compared using a ROC curve with mortality after 28 days of hospital discharge. Results: Age, race and gender were included in the analysis as index variables of predisposition
\end{abstract}


$(\mathrm{P})$, the site of infection (I) levels of leukocytes and PCR (R) as the response variable, and classification of sepsis, severe sepsis and septic shock as an outcome variable (0). Conclusions: The scale based on the PIRO concept applied to Colombian population presents scores related to an area under the ROC curve above 0.70 which acceptably classifies patients with high mortality risk.

Keywords: Sepsis; infection; validity; septic shock. (Source: DeCS, Bireme).

\section{Introducción}

Sepsis es la principal causa de mortalidad en cuidado intensivo, y afecta a millones de personas en el mundo cada año ${ }^{1}$. El sistema PIRO es una herramienta teórica propuesta para estratificar los pacientes y fue recomendada por la Conferencia de Consenso de 2001 como una alternativa práctica para reemplazar la clasificación actual ${ }^{2}$. Aunque ha sido difícil extrapolar a la práctica clínica, varios estudios han evaluado este concepto en pacientes atendidos en Unidad de Cuidado Intensivo y más recientemente se ha intentado validar en población ingresada en el servicio de urgencias, adicionalmente, estudios sobre el valor pronóstico del concepto PIRO requiere de evaluaciones en diferentes poblaciones para explorar su utilidad como herramienta de estratificación de riesgo ${ }^{3}$.

El objetivo del estudio fue diseñar y validar una escala de pronóstico basada en el concepto PIRO de los pacientes ingresados por sepsis en una población colombiana.

\section{Materiales y métodos}

En estudio de cohorte prospectiva se analizaron 559 pacientes con diagnóstico de sepsis quienes fueron seleccionados al ingreso al servicio de urgencias de una institución de cuarto nivel de complejidad con diagnóstico de sepsis, adicionalmente se solicitó el consentimiento informado al paciente o algún familiar con capacidad legal para otorgarlo, posterior a esto cada participante fue evaluado de acuerdo con los criterios de severidad propuestos en la conferencia de 2001, y asignados a una de tres categorías: sepsis, sepsis severa o choque séptico.
Se realizó una medición basal de las escalas APACHE II, Escala de Charlson, Escala SOFA y se tomaron los valores de leucocitos informados en la historia clínica, adicionalmente se midieron los niveles de PCR durante el primer día de ingreso.

\section{Tamaño de la muestra}

El estudio incluyó a 559 participantes quienes cumplieron criterios y se encontraban con datos completos en la base de datos para el momento de la realización del estudio. El seguimiento a los 28 días del ingreso a la institución fue realizado mediante llamada telefónica y los eventos de mortalidad fueron verificados con los familiares mediante la revisión de los certificados de defunción e historia clínica.

\section{Análisis estadístico}

Se realizó un análisis descriptivo mediante promedios y desviación estándar, mediana y rango intercuartil en el caso de las variables no paramétricas. Se realizó un análisis log binomial de cada una de las variables propuestas mediante el concepto PIRO con la mortalidad a 28 días como variable de desenlace. Se establecieron Riesgos relativos, intervalos de confianza del 95\% para cada una de las variables, considerando los valores de p significativos menores a 0.05 . Se realizaron curvas ROC para establecer los puntos de corte y definir los puntajes de la nueva escala.

Para cada ítem se estableció un puntaje de 0 a 3 de acuerdo con el resultado del análisis multivariado y los RR encontrados, finalmente se sumaron los puntajes para obtener un puntaje total que fue contrastado nuevamente con el desenlace a los 28 días mediante una curva ROC y sus intervalos de confianza del 95\%. Los análisis fueron realizados en el Software STATA versión 11. 


\section{Consideraciones éticas}

Este estudio fue aprobado por el Comité de Ética de la Universidad Autónoma de BucaramangaColombia.

\section{Resultados}

Los pacientes incluidos en el análisis fueron captados en el periodo de tiempo de 2011-2013 y se encontró que se presentaron diferencias en los promedios de edad, menor edad para los sobrevivientes en relación con los no sobrevivientes, los hombres presentaron una mayor mortalidad, aunque esta diferencia no fue estadísticamente significativa, los puntajes obtenidos en la escala de Charlson fue dos puntos mayor en el grupo de no sobrevivientes en relación con los sobrevivientes, la escala apache II, sofá y la medición de PCR fueron diferentes en los dos grupos con valores de $\mathrm{p}<0.05$, los valores de leucocitos fueron similares en ambos grupos. (Tabla 1)

En el análisis log binomial se observaron asociaciones estadísticamente significativas con las variables edad, puntaje de la escala de Charlson, sitio de infección, PCR y la clasificación de sepsis. (Tabla 2)
Se seleccionaron los puntos de corte con mayor sensibilidad y especificidad y basados en ellos se definieron puntajes para la edad $>=65$ años $\mathrm{y}<65$ años, con puntajes de uno y cero respectivamente, para la escala Charlson los puntos de corte son $>=3$ y $<3$, con puntajes de uno y cero respectivamente, los sitios de infección se calificaron de uno a cuatro considerando las asociaciones urinario, pulmonar, abdominal e infección del torrente sanguíneo, respectivamente. Los valores de PCR fueron divididos con los puntos de corte $<15$ y mayores de $15 \mathrm{mg} / \mathrm{dl}$ con valores de cero y uno respectivamente, así como la clasificación de severidad con puntajes de cero, uno y dos. (Tabla 2)

Tabla 1. Características de la población de estudio

\begin{tabular}{lrrr}
\hline Variable & \multicolumn{1}{c}{ Vivos } & \multicolumn{1}{c}{$\begin{array}{c}\text { No } \\
\text { sobrevivientes }\end{array}$} & $\begin{array}{c}\text { Valor } \\
\text { de p }\end{array}$ \\
\hline Edad (años) & $55.43(18.63)$ & $68.64(19.03)$ & 0.0000 \\
Sexo, masculino & $34(12.50)$ & $238(87.50)$ & 0.61 \\
Charlson, media & $1.57(1.78)$ & $3.37(3.19)$ & 0.0000 \\
$\begin{array}{l}\text { DE } \\
\text { Apache, medida }\end{array}$ & $9.08(5.61)$ & $17.87(8.61)$ & 0.0000 \\
$\begin{array}{l}\text { DE } \\
\text { Sofa, media DE }\end{array}$ & $1.50(2.17)$ & $5.28(4.22)$ & 0.0000 \\
PCR, media DE & $96.30(84.96)$ & $74.29(65.69)$ & 0.03 \\
$\begin{array}{l}\text { Leucocitos, } \\
\text { media DE }\end{array}$ & $16972(8408)$ & $16125(7116)$ & 0.4142 \\
\hline
\end{tabular}

Tabla 2. Variables categorizadas según el sistema PIRO y su asociación con mortalidad, expresadas como riesgo relativo (RR) e intervalo de confianza del (IC95\%)

\begin{tabular}{|c|c|c|c|c|c|}
\hline & & $\mathbf{R R}$ & IC95\% & Valor de p & Puntaje en la escala PIRO \\
\hline \multirow[t]{3}{*}{$P$} & Edad & 1.03 & $1.02-1.05$ & 0.000 & $\begin{array}{l}>=65 \text { años }(1) \\
<65 \text { años }(0)\end{array}$ \\
\hline & Raza & 1.09 & $0.59-2.01$ & 0.75 & $(0)$ \\
\hline & Género, masculino & 0.80 & $0.53-1.20$ & 0.28 & $(0)$ \\
\hline \multirow[t]{3}{*}{ I } & Puntaje obtenido en la Escala de Charlson & 1.29 & $1.26-1.33$ & 0.000 & $\begin{array}{l}<3 \text { puntos }(0) \\
>=3 \text { puntos }(1)\end{array}$ \\
\hline & Urinario* & 1 & & & $(1)$ \\
\hline & Pulmonar & 2.20 & $1.22-3.95$ & 0.008 & (2) \\
\hline \multirow[t]{4}{*}{$\mathrm{R}$} & Abdominal & 3.34 & $1.71-6.55$ & 0.000 & (3) \\
\hline & Infección del torrente sanguíneo & 5.3 & 1.77- 15.79 & 0.003 & (4) \\
\hline & Leucocitos & 0.99 & 0.99-1.0 & 0.42 & $(0)$ \\
\hline & PCR ng/dl & 0.99 & $0.99-0.99$ & 0.04 & $\begin{array}{r}<15 \mathrm{ng} / \mathrm{dl}(0) \\
>=15 \mathrm{ng} / \mathrm{dl}(1)\end{array}$ \\
\hline \multirow[t]{3}{*}{0} & Sepsis & 1 & & & $(0)$ \\
\hline & Severa & 4.85 & $2.08-11.28$ & 0.000 & (1) \\
\hline & Choque & 23.33 & $11.48-47.39$ & 0.000 & (2) \\
\hline
\end{tabular}

*Los otros sitios de infección en el modelo log binomial no presentaron valores significativos. 
Los puntajes de la escala muestran que el puntaje mayor a 3 clasifica correctamente a más del $70 \%$ de los participantes, esto se apoya con el LR positivo, el cual muestra que es dos veces más frecuente el hallazgo en los puntajes mayor de $3 \mathrm{y}$ el LR negativo que informa que el hallazgo es 5 veces más frecuente en quienes no tienen la enfermedad.
Más de 5 puntos en la escala propuesta tienen el mejor LR positivo de los demás puntos de corte analizados. (Tabla 3) La totalidad de los puntajes obtenidos mediante la escala fueron contrastados mediante una curva ROC el área bajo la curva fue de 0.75 IC95\%( 0.70-0.80). (Gráfico 1)

Tabla 3. Análisis de sensibilidad y especificidad de la escala concepto PIRO

\begin{tabular}{|c|c|c|c|c|c|}
\hline Punto de corte & Sensibilidad & Especificidad & Correctamente clasificado & LR positivo & LR negativo \\
\hline$>=0$ & $100 \%$ & $0 \%$ & $13.24 \%$ & 1.0000 & \\
\hline$>=1$ & $100 \%$ & $25.36 \%$ & $35.24 \%$ & 1.3398 & 0.0000 \\
\hline$>=2$ & $90.5 \%$ & $47.01 \%$ & $52.77 \%$ & 1.7086 & 0.2012 \\
\hline$>=3$ & $60.8 \%$ & $73.81 \%$ & $72.09 \%$ & 2.3223 & 0.5309 \\
\hline$>=4$ & $33.78 \%$ & $90.72 \%$ & $83.18 \%$ & 3.6411 & 0.7299 \\
\hline$>=5$ & $5.41 \%$ & $98.97 \%$ & $86.58 \%$ & 5.2432 & 0.9558 \\
\hline$>=6$ & $0 \%$ & $99.7 \%$ & $86.58 \%$ & 0.0000 & 1.0021 \\
\hline$>6$ & $0 \%$ & $100 \%$ & $86.76 \%$ & & 1.0000 \\
\hline
\end{tabular}

\section{Gráfica 1. Curva ROC puntaje total concepto PIRO y la mortalidad a los 28 días del ingreso}

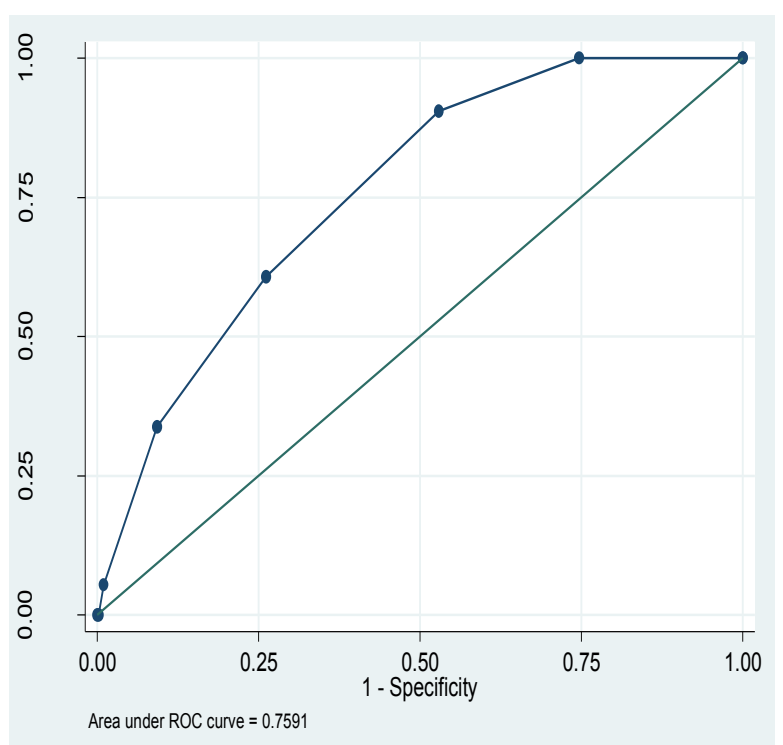

\section{Discusión}

Las escalas basadas en el concepto PIRO han sido evaluadas en diferentes poblaciones han mostrado ser útiles para establecer el pronóstico de la sepsis, el presente artículo muestra como con una serie de ítems orientados mediante este concepto es posible clasificar a los pacientes y establecer la probabilidad de mortalidad.
Yun-Xia Chen y cols., analizaron en una escala basada en este concepto, variables como la edad, la presentación de enfermedad pulmonar obstructiva crónica, la presencia de enfermedad hepática, residencia en un hogar de cuidado y malignidad como variables ubicadas en la predisposición, la infección en piel, cualquier otro sitio de infección y neumonía en la categoría de Infección, frecuencia respiratoria, frecuencia cardiaca y porcentaje de bandas en la categoría de respuesta, presión sistólica, BUN, falla respiratoria o hipoxemia, lactato sérico y conteo de plaquetas como variables del modelo en relación con la disfunción de órganos. La escala formulada por Yua- Xia chen, pudo demostrar mayor capacidad discriminativa en relación con otras escalas como la escala MEDS, APACHE II y SOFA 4 .

S. Tafelsky et al., realizaron un estudio de comparación de tres escalas basadas en el concepto PIRO, en doscientos setenta y ocho pacientes sépticos, incluyó puntajes propuestos por Moreno, Rubulotta y Howell et al., encontrando valores de área ROC entre 0.74, 0.64 y 0.75 respectivamente, para cada uno de los modelos propuestos, reconocen como limitación de su estudio la no inclusión de variantes genéticas y limitada a fenotipos. La capacidad discriminativa es denominada como buena pero 
sin suficiente calibración ${ }^{5}$. Cardoso y cols., elaboraron una escala basada en el concepto PIRO evidenciando la utilidad de este abordaje teórico en la inclusión de pacientes en estudios clínicos, en la definición de los criterios de inclusión y en el manejo de pacientes con infección ${ }^{6}$.

Granja y cols., identificaron como factores para el componente $\mathrm{P}$ el género, la edad, falla hepática crónica, falla renal crónica y cáncer metastásico, para el componente I cultivos de sangres positivos, terapia de antibióticos en concordancia con las guías, y sepsis asociada al cuidado de la salud. Para el componente $\mathrm{R}$, la pendiente de la proteína $C$ reactiva, la frecuencia cardiaca en el día 1, la pendiente de la frecuencia cardiaca, la pendiente de los neutrófilos, para el componente 0 fueron el lactato sérico en el día 1, la pendiente del lactato sérico, el puntaje SOFA del día 1, y la pendiente del puntaje SOFA. El área bajo la curva ROC fue de 0.83 IC95\%(0.81-0.87)7.

Marshall, propone que el modelo PIRO se aplique en el diseño de un programa de investigación clínica, adicionalmente recomienda que más allá de incorporar el modelo PIRO en el diseño de futuras investigaciones, es necesario ensamblar cohortes que expliquen el curso clínico de la enfermedad, de tal manera que permita modificar la respuesta a la intervención clínica inicial ${ }^{3}$.

En conclusion la escala basada en el concepto PIRO aplicada a población colombiana, presenta puntajes relacionados con una área bajo la curva ROC de 0.75 , lo cual la clasifica como una escala aceptable para evaluar a los pacientes con riesgo de mortalidad.

Financiación: El presente estudio fue financiado mediante la convocatoria interna de investigaciones 2013-2014 de la Universidad Autónoma de Bucaramanga mediante el contrato 134074.

Agradecimientos: La autora agradece al Grupo de Investigación de Mediadores Inflamatorios y Enfermedad.

Conflicto de intereses: Ninguno declarado por la autora.

\section{Referencias}

1. Angus DC, Linde-Zwirble WT, Lidicker J, Clermont G, Carcillo J, Pinsky MR. Epidemiology of severe sepsis in the United States: analysis of incidence, outcome, and associated costs of care. Crit Care Med. 2001;29(7): 1303-10.

2. Levy MM, Fink MP, Marshall JC, Abraham E, Angus D, Cook D, et al. 2001 SCCM/ESICM/ACCP/ATS/SIS International Sepsis Definitions Conference. Crit Care Med. 2003;31(4):1250-6.

3. Marshall JC. The PIRO (predisposition, insult, response, organ dysfunction) model: toward a staging system for acute illness. Virulence. 2014;5(1):27-35.

4. Chen YX, Li CS. Risk stratification and prognostic performance of the predisposition, infection, response, and organ dysfunction (PIRO) scoring system in septic patients in the emergency department: a cohort study. Crit Care. 2014;18(2):R74.

5. Tafelski S, Nachtigall I, Stengel S, Wernecke K, Spies CD. Comparison of three models for sepsis patient discrimination according to PIRO: predisposition, infection, response and organ dysfunction. Minerva Anestesiol. 2014;81(3):264-71.

6. Cardoso T, Teixeira-Pinto A, Rodrigues PP, Aragão I, Costa-Pereira A, Sarmento AE. Predisposition, insult/infection, response and organ dysfunction (PIRO): a pilot clinical staging system for hospital mortality in patients with infection. PLoS One. 2013;8(7):e70806.

7. Granja C, Póvoa P, Lobo C, Teixeira-Pinto A, Carneiro A, Costa-Pereira A. The predisposition, infection, response and organ failure (Piro) sepsis classification system: results of hospital mortality using a novel concept and methodological approach. PLoS One. 2013;8(1):e53885. 